\title{
Driver Drowsiness Detection Methods: A Comprehensive survey
}

\author{
D. Venkata Subbaiah(Research Scholar) ${ }^{1}$, Prof. Prasad Reddy P.V.G.D ${ }^{2}$, Prof.K. Venkata Rao ${ }^{3}$ \\ Department of Computer Science \& Systems Engineering \\ Andhra University College of Engineering $(A)$ \\ Andhra University, Visakhapatnam
}

\begin{abstract}
Drowsy driving is one of the main causes for accidents on roads which leads to death. So, detection of fatigue of the driver and indicating it is an active research area. Most of the traditional methods followed either physiological, vehicle or behavioral based methods for drowsiness detection techniques. It is observed that some methods require sensors which are expensive while others are intrusive to the driver which distract the driving. Therefore, a real time driver's drowsiness detection system with low cost and high accuracy is an essential need. This paper presents different traditional methods used in drowsiness detection for over a decade. This study analyses different machine learning methods in drowsiness detection. It also reviews related studies in the period between 2008 and 2018 focusing on different methods used including latest machine learning techniques.
\end{abstract}

Keywords: Drowsy Driver, facial landmarks, Drowsiness Detection System, machine learning.

\section{INTRODUCTION:}

1.1 Drowsiness: Drowsiness is the state of the human body where the body craves for sleep. The main symptoms of being drowsy are the inability to keep the eyes open even for a short period of time. The state of drowsiness plays a very important role while a person is driving on the roads. Drivers may experience drowsiness as shown in the Fig. 1(source.[1]) not because of mental disorders, but because of lack of good sleep[1].

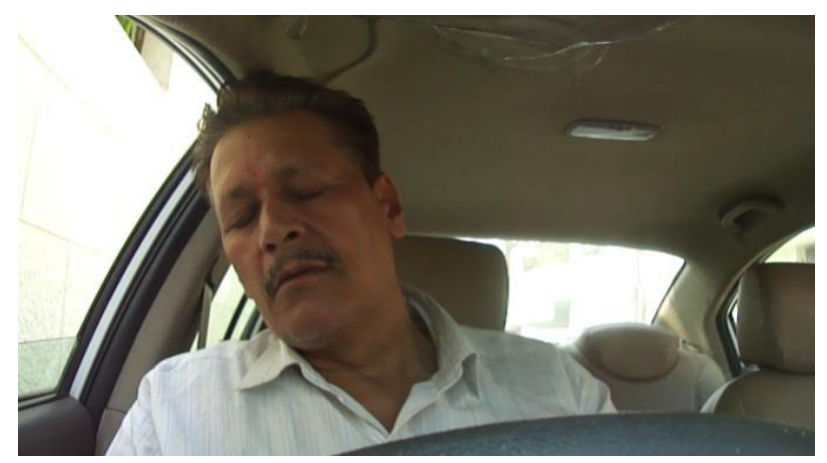

Fig.1 Driver Drowsy state.

1.2 Driver Fatigue and Road Accidents: Driver fatigue is the reason behind most of the road accidents every year. The actual number of sleep related accidents cannot be calculated but research and surveys carried out by organizations say that driver fatigue may be the contributing reason for up to $20 \%$ of the road accidents taking place. A person who is sleep deprived can't make decisions properly, because the brain needs to function and for the brain to function, it needs proper and adequate sleep which the drivers don't follow and continue prolonged driving without proper sleep. Drowsiness reduces the response time and this is a huge threat for secure and safe driving. Most crashes caused by tired drivers are most likely to happen on long journeys on monotonous roads in the late night hours between 1am and 4am because people feel that the roads will be deserted at that point of time in the night and they feel they can get along the way ignoring the possibility of accidents. There are various measures to determine the level of driver drowsiness. These measures are grouped as follows:
i. Physiological measures
ii. Vehicle-based measures
iii. Behavioral measures

i.physiological measures: physiological measures uses different electrodes such as Electroencephalography (EEG), Electrocardiography(ECG) and Electrooculogram (EOG). The raw data access by electrodes and pre process the data for artifact removal and filtering. After pre processing the data divided into different frequency bands. These frequencies can then be used to accurate measurement of drowsiness with detection of inner stage of driver. Although these approaches have proven relatively effective for examining 


\section{International Journal of Research in Advent Technology, Vol.7, No.3, March 2019 E-ISSN: 2321-9637 \\ Available online at www.ijrat.org}

physiological and cognitive states in humans, issues with wearability, have limited the feasibility of using these systems in real-world driving conditions[2-3].

ii.Vehicle-based measures: In this method, driver drowsiness measurement is determined in a simulated environment by placing sensors on various vehicle components, including the steering wheel and the acceleration pedal, then the received signals from the sensors are analyzed to determine the level of drowsiness[4]. Two most frequent measures are the steering wheel movement and the standard deviation of lane position. These methods are non-invasive, but may not be reliable in detecting drowsiness accurately because they are dependent on the nature of the road and the driver's driving skills.

iii.Behavioral measures: Facial expressions based drowsiness detection makes use of computer vision to detect and recognize the facial motion and changes in appearance during drowsiness[5]. It accepts a stream of input images from a camera in front of the driver and pass these images through the four main image processing stages: face detection and tracking, feature extraction, feature selection and classification.

a) Face Detection and tracking: This stage typically aims to detect the face in the image frames. The characteristics of a good detection algorithm are:

* Robust - very high detection rate (truepositive rate) \& very low false-positive rate always.

* Real time - For practical applications at least 2 frames per second must be processed.

* Face detection only (not recognition) Distinguish faces from non-faces only.

b) Feature extraction: In this phase, important features from the face detection are extracted that are utilized to comprehend the images easier.

c) Feature selection/Analysis: Extracted features can then be processed further, as is the case for eye state, head movement, blinking rate and yawning. After obtaining these features, further processing is applied to determine the level of drowsiness

d) Classification: Once the optimal features are selected, the next step is to classify the images of face for identifying the drowsiness. Therefore, implementing an appropriate classifier is very important. Each classifier includes two important modes: training and testing. Deep Neural Network (DNN), convolutional neural network (CNN), support vector machine (SVM), etc, are some of the machine learning classifiers used in drive drowsiness detection.

\section{RELATED WORK:}

Recent approaches for detecting driver drowsiness generally divided into three main categories: physiological measures, vehicle-based measures, and driver behavioral measures. Behavioral measures have the largest contribution last few years.

Hong Su et al. [6] described, a technique called Partial least square regression (PLSR) which monitors eyelid movement features and predicts drowsiness. It uses strong collinear relations among the eyelid movement features to predict drowsiness. The predictive precision of their model shows that it is a good method to use multi features together to detect the state of drowsiness.

Joshua M. Clanton et al. [7] proposed, a vision-based lane departure warning (LDW), which alerts the driver of an unintended lane departure. By working on vision sensors that detect lane markings on the roadway. This approach uses GPS, sensors and high accuracy map to assist the LDW system. There could be a position error due to low accuracy in GPS receiver and this may take too much time to locate a vehicle on the road. Hence they gave a method to measure the error.

Yong Du Peijun et al.[8], Proposed a visionbased method to detect the driver fatigue. In this method inter frame difference approach and mixed skin color model is used to decide whether the frame contains a face or not. From the face region this method simulating the crystallization process to segment the eyes. The eye status is analyzed based on eye area, average height of the pupil and width to height ratio. Finally, the driver fatigue is confirmed by analyzing the changes of eye states.

$\mathrm{Hu}$ Shuyan et al. [9] proposed drowsiness prediction using SVM. The features extracted from EOG data i.e two EOG vertical channels combined into one by averaging two signals. The extracted 11 features fed as input to the SVM drowsiness detection model. This model can detect three different drowsiness levels. The evaluated result shows that the drowsiness detection accuracy is quite high especially when the subjects are very sleepy. 


\section{International Journal of Research in Advent Technology, Vol.7, No.3, March 2019 E-ISSN: 2321-9637 \\ Available online at www.ijrat.org}

Antoine Picot et al.[10] proposed an algorithm for the automatic detection of drivers drowsiness based on visual features. The relevant visual features are selected by data-mining methods on consistent database. The fuzzy logic merged different features to detect the drowsiness. This algorithm evaluated on large number of drivers and validated with EOG signals. This system achieved $81.7 \%$ accuracy.

Aleksandra Kro'lak et al.[11] proposed vision-based eye-blink monitoring system for fatigue monitoring, human- computer interfacing and lie detection. This system initially performed face detection with Haar-like features method. Eye region extraction performed by geometric dependencies, eye blink detection performed by template matching. Finally eye-blink classification performed by pre defined threshold value. The system achieved 99\% accuracy and deployed as a open source software.

M.Hassaballa et al.[12], Proposed a technique to detect the eye centre point based on two facts. From the gray scale, image identify the unpredictable local intensity using entropy of eye regions and the centre of the eye region that is too dark circle with low intensity. A score based on the entropy of eye and darkness of iris used to detect the coordinates of the eye centre. This technique evaluated two datasets such as FERET and BioID and achieved detection rate 97.8\% for FERET dataset and 94.3\% for BioID. When combined the both datasets achieved the accuracy $96.2 \%$.

Mathew Sacco et al.[13] designed a Fatigue monitoring system, it consist of five stages. Initially pre processing the image using Histogram equalization and median filtering, detect the facial features using Viola-Jones Object detection framework, determine the feature state with template matching, features extracted by time duration between successive closed eye states frame, and finally classified the fatigue using SVM classification. This technique achieved accuracy of $95.2 \%$.

G. Kong et al. [14] designed a visual analysis of eye state and head pose (HP) for continuous monitoring of driver. This technique used visual features like eye index (EI), pupil activity (PA) and head pose (HP) to extract crucial information on nonalertness of the driver. SVM classifies a sequence of video segments into alert or non-alert events. It achieved high classification accuracy with low probability errors.
Yijia Sun et al.[15] proposed a technique which combines HMM and SVM to detect temporal dynamics of eye blinks. This model extracts the features for each frame from eye image sequences using HOG, Gabor filter, LBP, and optical flow and pixel intensity. Combined HMM and SVM used to detect eye blink' and calculates duration of eye blink. They also compared the technique with several popular feature extraction methods. Optical flow and pixel intensity feature extraction achieved highest accuracy per frame.

Eyosiyas et al. [16] proposed a HMM (hidden markov method) based dynamic model and is used in detection of driver drowsiness and distraction. Features extracted from the grayscale image, crop the driver face and eyes which are most likely located. Features are selected using adaboost weak learning algorithm. HMM used as a classification algorithm to detect drowsiness with simulated driving setup.

Leo Pauly, et al[17] presents a method of drowsiness detection for the images obtained using web cameras under normal lighting conditions. The drowsiness detection method uses Haar based cascade classifier for eye tracking and combination of Histogram of oriented gradient (HOG) features combined with Support Vector Machine (SVM) classifier for blink detection. Once the eye blinks are detected then the PERCLOS is calculated from it. If the PERCLOS value is greater than 6 seconds then the system is detected as drowsy. The system obtained an accuracy of $91.6 \%$.

Mr. Raees Ahmad,et al[18] implemented driver drowsiness detection system using cascade object identifier from vision toolbox of Matlab. It used face, eyes, nose and mouth from the image which is captured from web camera. This technique identified whether eyes are open or closed based on threshold value. From mouth portion yawning is determined. Results are formulated under different categories like normal driver, driver with glass under different lighting conditions.

B. Mandal, L. Li,et al.[19] proposed vision based method to detect the fatigue of driver. The system detects the head-shoulders of the image, followed by face and eye detections and eye openness estimation. Finally a multi model fusion scheme designed infers eye state and a PERCLOS measure on the continuous measure of eye openness to predict driver's attention state. The designed method 


\section{International Journal of Research in Advent Technology, Vol.7, No.3, March 2019 E-ISSN: 2321-9637 \\ Available online at www.ijrat.org}

effectively monitors driver's attention without extra requirements for cameras.

Nur Fatin Izzati y et al.[20] proposed a technique to determine the height of the iris, and classify whether the eye is opened or closed state. Viola jones algorithm used to detect the face and eyes, and Kenada Lukas Tomasi(KLT) algorithm used for tracking the features of face and eyes. The classification based on height of the iris is localized. The performance of the algorithm is tested on Zhejiang University (ZJU) Database and achieved successful results is identify the height of the iris.

Chan-Hee Jeong, et al.[21] proposed Discriminative Bayesian active shape model(ASM) by incorporating the Extreme pose case named as poseextended active shape model(PE-ASM). This model includes the HMM to detect facial features which are drowsy or non-drowsy. This technique evaluated on two facial databases such as Boston university face database and their custom-made driving database. This technique outperforms AAM and ASM.

Fang Zhang et al. [22] proposed an Eye state recognition method based on Convolution Neural Network (CNN), to solve state of the eye problems due to eye glasses. This technique used AdaBoost Algorithm to detect face, eyes and calculate the percentage of eyelid closure (PERCLOS), blink frequency to detect fatigue. This method is robust and has high accuracy even driver wear the glasses.

D. Panicker et al.[23] proposed a drowsiness detection system in three stages. The first stage was face detection using elliptical approximation and template matching techniques. In the second stage, the open eye is detected using the proposed iris-sclera pattern analysis(ISPA) method. In the third stage, the drowsiness state of the driver was determined using PERCLOS measure. The entire system was designed to be independent of any specific data sets for face or eye detection.

Farnaz Abtahi, et al.[24] proposed an EAC Net(Enhancing and Cropping Net) that is combination of E-Net and C-Net for facial action unit(FAU) detection. E-Net is to force the neural network to pay more attention to enhance learning FAU regions on face images. C-Net ensures that the network learned features by cropping layers in aligned facial area. With deep pre-trained model to focus on interest regions, this approach shows its power in FAU detection on multiple datasets
Jie Lyu et al. [25] proposed, a method "long term multi granularity deep framework" to detect drowsiness which includes two key components: First, multi granular convolutional neural network (MCNN) which uses CNN extractors on facial patches and extracts facial representation for large variation of head pose. Second a deep long short term memory network is applied on facial representation and distinguishes blinking and closing eyes. This approach achieved $90.05 \%$ accuracy for $37 \mathrm{fps}$ speed on the evaluation set of the public NTHU-DDD dataset.(c)

Ashish Kumar et al. [26] developed a lowcost real-time driver drowsiness detection system. Driver's face detected in each frame from the webcam recorded video, using image processing techniques. The features are identified as eye aspect ratio(EAR), mouth opening ratio(MOR), and nose length ratio(NLR). The SVM classification method is used for classification of drowsiness. This technique achieved sensitivity $95.58 \%$ and specificity $100 \%$.

Herleeyandi Markoni et al. [27] proposed a hybrid method combined convolutional neural network (CNN) and long short-term memory (LSTM) for handling the real-time driver drowsiness detection. The MTCNN extract face and benchmark features with P-Net, R-Net and O-Net. LSTM provide a learnable parameter and adopt the changes for robustness. This method defined time skip combination LSTM(TSC LSTM) and used as a classification algorithm for drowsiness detection.

Eddie E. Galarza1 et al.[28] designed the human computer interaction Drowsiness detection system with a mobile application with android OS. This system consists of pattern analysis, analysis of eye and facial expression, and physiological signal analysis. Pattern analysis analyzed road lines and pressure on steering wheel, eye and facial expression analysis done under the natural and infrared lighting and finally physiological signal analysis analyzed the EEG and heart rate signals. This android detection system achieved accuracy of $93.37 \%$.

\section{FINDINGS OF THE LITERATURE SURVEY}

The article discussed about different Techniques utilized by various authors in their research works. From the above literature review, It is concluded that most of the researchers used classifier approaches. For this, they used a classification algorithm like Support Vector Machine (SVM), Convolutional neural networks and hidden Markov models in the context of 


\section{International Journal of Research in Advent Technology, Vol.7, No.3, March 2019 E-ISSN: 2321-9637 \\ Available online at $w w w . i j r a t . o r g$}

drowsiness detection. The analysis reveals that support vector machine is the commonly used technique, but Convolutional neural networks performed better than the other techniques. Careful analysis of the literature concludes the following points:

- If there is any reflective object found behind the driver, accurate results cannot be produced.

- $\quad$ Some of the Machine learning techniques such as SVM, CNN and HMM are studied. As there are limited no. of standardized datasets, it is very difficult to compare these approaches.

- For enhancing the accuracy of drowsiness classification, an efficient dimensionality reduction approach and a combination of both high-level and low-level features are to be used.

- For enhancing the classification performance of drowsiness detection in a complex background, a multi-objective machine learning classifier is to be designed.

- The state of eyes of the driver can be used to detect the tiredness but that would be affected by wearing sunglasses.

- For the drivers who are wearing glasses and are under varying light conditions, camera should be improved, mounting and image processing algorithms should be more robust.

- For the better estimation of the blinking velocity, higher sampling frequency is to be used.

- Inorder to classify the face features from the images acquired from the standard dataset for identifying drowsiness, an effective face detection technique is to be designed.

- Improvement and validation of the algorithms is based on the currently conducted large real road study.

- For analysis of eyes, the amount of time the eyes are closed indicates whether the driver is falling asleep or not. This together with other information such as eye texture will be combined with the present head posture to provide a full driver monitoring system.

Limitations: As observed from the existing Drowsiness Detection Systems, the following factors affects in the detection of drowsiness and in obtaining accuracy.

- The movement of the camera shows a great impact as it varies at certain points (Speed brakers) on the roads.

- The lighting conditions usually vary depending on the time of that particular moment.
- The performance is greatly affected by the frame rate used to capture images of the driver's face.

\section{CONCLUSION}

The survey of literate indication survey Physiological measures requires sensors which are more expensive and vehicle based methods may not be reliable because of their practical limitations. In order to build an efficient system to detect drowsiness, behavioral measures it is better to obtain the information with help of cameras to detect slight changes in driver's facial expressions. The fundamental working principle is to detect abnormality in the driving pattern. Extract the discriminative features using dimensionality reduction algorithms.

It's recommended to use feature extraction followed by classification as a hybrid approach to obtain high accuracy of drowsiness detection.

\section{REFERENCES}

[1] Chisty, Jasmeen Gill A Review: Driver Drowsiness Detection System", IJCST - Volume 3 Issue 4, Jul-Aug 2015, pp. 243-252 ISSN: 23478578.

[2] Karamjeet Singh,Rupinder Kaur,'Physical and Physiological Drowsiness Detection Methods", IJIEASR, pp.35-43,vol.2,2013.

[3]. AntoinePicot,SylvieCharbonnier,'On-Line Detection of Drowsiness Using Brain and Visual Information",IEEE Transaction on systems, man and cybernetics part a: systems and humans, VOL. 42, NO. 3,2012

[4]. A. D. McDonald, C. Schwarz, J. D. Lee, and T. L. Brown, "Real- Time Detection of Drowsiness Related Lane Departures Using Steering Wheel Angle," Proc. Hum. Factors Ergon. Soc. Annu. Meet., vol. 56, no. 1, pp. 2201-2205, 2012.

[5]. S. Ghosh, T. Nandy, and N. Manna, "Real Time Eye Detection and Tracking Method for Driver Assistance System," Adv. Med. Electron., 2015.

[6] Hong Su and Gangtie Zheng, "A Partial Least Squares Regression-Based Fusion Model for Predicting the Trend in Drowsiness" IEEE TRANSACTIONS ON SYSTEMS, MAN, AND CYBERNETICS-PART A: SYSTEMS AND HUMANS, VOL. 38, NO. 5, SEPTEMBER 2008.

[7] J. M. Clanton, D. M. Bevly, and A. S. Hodel, "A low-cost solution for an integrated multisensor lane departure warning system," IEEE Trans. Intell. Transp. Syst., vol. 10, no. 1, pp. 47-59, Mar. 2009. 


\section{International Journal of Research in Advent Technology, Vol.7, No.3, March 2019 E-ISSN: 2321-9637 \\ Available online at www.ijrat.org}

[8]. Yong Du Peijun Ma Xiaohong $\mathrm{Su}$ Yingjun Zhang, "Driver Fatigue Detection based on Eye State Analysis" , Proceedings of the 11th Joint Conference on Information Sciences (2008).

[9]. Hu Shuyan *, Zheng Gangtie," Driver drowsiness detection with eyelid related parameters by Support Vector Machine", Expert Systems with Applications 36 (2009) 7651-7658, 0957-4174/\$ see front matter _ 2008 Elsevier Ltd. All rights reserved.

[10]. Antoine Picot, Sylvie Charbonnier and Alice Caplier, "Drowsiness detection based on visual signs: blinking analysis based on high frame rate video", 978-1-4244-2833-5/10/\$25.00 @2010 IEEE.

[11]. Aleksandra Kro'lak - Paweł Strumiłł, "Eyeblink detection system for human-computer interaction", Published online: 2 October 2011 The Author(s) 2011. This article is published with open access at Springerlink.com

[12]. M.Hassaballa, Kenji Murakami, Shun Udo, “An Automatic eye Detection method for Gray Intensity Faial images “, IJCSI, Vol. 8, Issue 4, No 2 July 2011, pp. 272-282.

[13]. Matthew Sacco, reuben A. Farrugia, " Driver fatigue monitoring system using support vector Machine “, 978-1-4673-0276-0, 2012, IEEE.

[14] Ralph Oyini Mbouna, Seong G. Kong, Senior Member, IEEE, and Myung-Geun Chun," Visual Analysis of Eye State and Head Pose for Driver Alertness Monitoring." IEEE TRANSACTIONS ON INTELLIGENT TRANSPORTATION SYSTEMS, VOL. 14, NO. 3, SEPTEMBER 2013

[15]. Yijia Sun, Stefanos Zafeiriou, Maja Pantic, "A Hybrid System for On-line Blink Detection", 2013.

[16].E. Tadesse, W. Sheng and M. Liu, "Driver drowsiness detection through HMM based dynamic modeling," 2014 IEEE International Conference on Robotics and Automation (ICRA), Hong Kong, 2014, pp. 4003-4008

[17]. Leo Pauly, Deepa Sankar," Detection of Drowsiness based on HOG features and

SVM classifiers", 2015 IEEE (ICRCICN), pp: 181186.

[18]. Mr. Raees Ahmad, Prof. J.N. Borole ," Drowsy Driver Identification Using Eye Blink

Detection ",Raees Ahmad et al, / (IJCSIT) International Journal of Computer Science and
Information Technologies, Vol. 6 (1) , 2015, 270274.

[19] B. Mandal, L. Li, G. S. Wang, and J. Lin, "Towards Detection of Bus Driver Fatigue Based on Robust Visual Analysis of Eye State," IEEE Trans. Intell. Transp. Syst., vol. PP, no. 99, pp. 113, 2016.

[20]. Nur Fatin Izzati y., M.M.Ibrahim, N.A.Manap, nur Shazwani A., " Analysis of eye closure duration based on height of Iris “, $20166^{\text {th }}$ International Conference on control system, Malaysia.

[21].In-Ho Choi, Chan-Hee Jeong and Yong-Guk Kim, ” Tracking a Driver's Face against Extreme Head Poses and Inference of Drowsiness Using a Hidden Markov Model”, Appl. Sci. 2016, 6, 137; doi:10.3390/app6050137.

[22] Fang Zhang et.al. ,'Driver Fatigue Detection Based on Eye State Recognition", International Conference on Machine Vision and Information Technology, 978-1-5090-4993-6/17 \$31.00 (C) 2017 IEEE, pp.-105-110.

[23] A. D. Panicker, and M. S. Nair. "Open-eye detection using iris-sclera pattern analysis for driver drowsiness detection." Sädhanā 42.11 (2017): 1835-1849.

[24]. Wei Li1 Farnaz Abtahi2 Zhigang Zhu1'2 Lijun Yin3, "EAC-Net: A Region-based Deep Enhancing and Cropping Approach for Facial Action Unit Detection", 978-1-5090-4023-0/ (C) 2017 IEEE, pp: 103-110

[25] Jie Lyu et. Al. , "Long-term Multi-granularity Deep Framework for Driver Drowsiness Detection", arXiv:1801.02325v1 [cs.CV] 8 Jan 2018.

[26] Ashish Kumar et. Al. "Driver Drowsiness Monitoring System using Visual Behavior and Machine Learning”, 978-1-5386-3527-8/18 2018 IEEE, pp.-339-344.

[27] J. M. Guo, and Herleeyandi Markoni. "Driver drowsiness detection using hybrid convolutional neural network and long short-term memory." Multimedia Tools and Applications (2018): 1-29.

[28]. Eddie E. Galarza1,1, Fabricio D. Egas1, Franklin M. Silva1, Paola M. Velasco1, Eddie D. Galarza1," Real Time Driver Drowsiness Detection Based on Driver's Face Image Behavior Using a System of Human Computer Interaction Implemented in a Smartphone",IEEE, 2018. 